Check for updates

Cite this: Mater. Adv., 2020, 1, 2897

Received 29th May 2020,

Accepted 24th June 2020

DOI: $10.1039 / \mathrm{d} 0 \mathrm{ma} 00362 \mathrm{j}$

rsc.li/materials-advances

\section{Spectral characteristics upon harvesting plasmonic hot electrons at the $\mathrm{Ag} / \mathrm{ZnO}$ heteromicrostructures $\uparrow$}

\author{
Dewan S. Rahman, ${ }^{a}$ Sudip Kumar Pal, ${ }^{a}$ Shib Shankar Singha, ${ }^{b}$ Susmita Kundu, \\ Soumen Basu (iD *de and Sujit Kumar Ghosh (iD *a
}

\begin{abstract}
Silver/zinc oxide heterostructures have been adopted to investigate the spectral properties that are of paramount importance to demonstrate the feasibility of band gap engineering upon injection of hot electrons in the ensuing microstructural architectures. Simultaneous hydrolysis of the precursor salts of variable compositions in a water/o-xylene binary solvent mixture under alkaline conditions provides a rational approach to induce co-organisation into a series of $\mathrm{Ag} / \mathrm{ZnO}$ microstructures. The generation of hot electrons in plasmonic silver particles upon illumination with light and their subsequent injection into $\mathrm{ZnO}$ provide unique opportunities for the manipulation of electromagnetic waves in the metal/ semiconductor heterostructures. Metallic colloids of silver have large absorption cross-sections and therefore, possess the ability to concentrate electromagnetic radiation in the subwavelength dimension and on a femtosecond time scale. Plasmonic excitation of silver nanostructures initiates a variety of photophysical processes, including non-radiative decay which produces non-thermal 'hot' carriers that can be injected along the vicinal semiconductor boundary harnessing the absorption loss. It has been revealed that a mere variation in the experimental conditions creates a diversity in the morphology that imbues considerable differences in the spectral characteristics of the $\mathrm{Ag} / \mathrm{ZnO}$ heterostructures. Since numerous metal nanoparticles are incorporated inside the giant semiconductor architecture, the obtained microstructures provide new model systems for the modification of respective theoretical models with many body electron transfer processes.
\end{abstract}

\section{Introduction}

The generation of plasmonic hot carriers upon excitation of metallic nanostructures and subsequent injection into semiconductors is critical in the transduction of light into electrical and chemical energy that renders important consequences in light harvesting processes. ${ }^{1-4}$ Modification of the electrostatic potential upon rectifying metallic contacts on semiconductors surfaces dates back to the proposition of the classic model by

\footnotetext{
${ }^{a}$ Department of Chemistry, Assam University, Silchar-788011, India.

E-mail: sujit.kumar.ghosh@aus.ac.in

${ }^{b}$ Department of Physics, Brahmananda Keshab Chandra College, 111/2 B. T. Road, Kolkata - 700108, India

${ }^{c}$ Sensor and Actuator Division, CSIR-Central Glass \& Ceramic Research Institute 196, Raja S.C. Mullick Road, Kolkata - 700 032, India

${ }^{d}$ School of Chemistry and Biochemistry, India

${ }^{e}$ TIET-Virginia Tech Center of Excellence in Emerging Materials, Thapar Institute of Engineering and Technology, Patiala-147004, India.

E-mail: soumen.basu@thapar.edu

$\dagger$ Electronic supplementary information (ESI) available. See DOI: 10.1039/ d0ma00362j
}

Schottky predicting the phenomenological boundary conditions for metal-semiconductor interfaces. ${ }^{5}$ The injection of plasmonic hot electrons in metal-semiconductor heterostructures opens opportunities to increase the spectral range of photon energies covering a substantial part of the solar spectrum and such photoinduced charge transfer processes can be harnessed in the context of solar energy conversion, plasmon enhanced photocatalysis, surface photochemistry, second harmonic generation, Schottky diodes, Fabry-Perot interference fringes and photoelectron microscopy. ${ }^{6}$ The physical origin of the light absorption by metal nanoparticles is the time-varying electric field of the electromagnetic radiation that causes collective oscillation of the conduction electrons in metal nanoparticles with a resonance frequency, often referred to as localised surface plasmon resonance (LSPR). ${ }^{7}$ The creation of an electric dipole in the metallic nanostructure upon light illumination is instantaneous and therefore the electric charge can redistribute across an interface on the time scale of the dephasing of the optically excited coherence. ${ }^{8}$ The confined surface plasmons, thus, after a short time, start to decay into reemitted photons through a radiative process or interband or 
intraband transitions via a non-radiative pathway, creating hot electron-hole pairs through Landau damping on the time scale of, typically, less than ten femtoseconds. ${ }^{9}$ The plasmonic hot electrons can further undergo thermalisation through electron-electron and electron-phonon scattering leading to the transduction of light, eventually, becoming transformed into thermal energy. ${ }^{10}$ However, the energetic hot electrons with photon energies lower than the semiconductor band gap can be captured before thermalisation by an adjacent semiconductor opening a new avenue for additional energy harvesting. ${ }^{11}$ The charge transfer across the interface can also occur by coherent coupling at the donor and acceptor states. The hot electrons, being confined at the subwavelength dimension prior to losing the energy can interact several times with the boundary which increases the probability of injection. The photogeneration of hot electrons in a metallic structure and their injection into a semiconductor is critical for the conversion of sub-band gap photons into electrical charges and therefore, to govern the photophysical, photochemical and photocatalytic aspects of the metal-semiconductor heterostructures. $^{12}$ The efficiency of the generation of hot carriers and subsequent injection due to carrier confinement and surface scattering is strongly dependent upon the composition and morphology of the individual components forming the metal-semiconductor heterostructures.

The recent advent of the art of nanomaterial synthesis and the design of heterostructures by controlled miniaturisation of two or more disparate materials, represents a potential approach for achieving advanced materials that has become more and more indispensable to fulfill the academic and industrial perspectives. ${ }^{13,14}$ The development of synthetic approaches offering a high degree of control over the composition and morphology using a wide range of material combinations has become an effective strategy for inducing new physicochemical properties. ${ }^{15}$ Zinc oxide ( $\left.\mathrm{ZnO}\right)$ has received considerable interest as a compound metal oxide semiconductor derived from the group IIb-VI series in the periodic table with the characteristics of a wide direct band gap $(3.37 \mathrm{eV})$, large exciton binding energy $(60 \mathrm{meV})$ at room temperature, good thermal and chemical stability, and low cost and environmental friendliness due to its biocompatibility. ${ }^{16}$ In addition to its numerous inherent physicochemical properties and diverse applications, ZnO displays high optical transparency and luminescence properties in the near UV-vis region and excellent electrical characteristics, suitable for making sensing devices. ${ }^{17}$ Silver particles, in the nanometer size regime, exhibit distinctive optical properties as the dielectric constant of the metal near the Fröhlich frequency gives rise to intense plasmon absorption in the visible region. ${ }^{18}$ It has been established that pure $\mathrm{ZnO}$ nanostructures exhibit weak optoelectronic features arising due to point defects, such as oxygen vacancy or interstitial $\mathrm{Zn}$ and these factors limit their utilisation directly to industrial applications. ${ }^{19}$ Therefore, doping of suitable substances into $\mathrm{ZnO}$ is indispensable for engineering of the electronic, optical, magnetic and transport properties. In addition, appropriate $\mathrm{n}$ - and p-type states are necessary in order to manufacture convenient optoelectronic devices. Achieving p-type doping in $\mathrm{ZnO}$ nanostructures has, often, been relatively difficult; amongst the elements of group IB, silver has been found to be the best-suited candidate due to its high solubility, larger ionic size, minimum orbital energy and ability to form shallower acceptor levels. ${ }^{20}$ It has been predicted that under O-rich conditions, the $\mathrm{Ag}$ atoms occupy $\mathrm{Zn}$ lattice sites forming AgZn defects. Doping of silver in $\mathrm{ZnO}$ provides the impurity band in the energy gap that could be ascribed to the formation of the $p$-type in these substances; the reduction in the energy gap leads to an increase in the efficiency that makes the utilisation of these materials in optoelectronic devices feasible. ${ }^{21}$ Moreover, due to the large $\mathrm{p}-\mathrm{d}$ coupling arising from the overlap between the $\mathrm{Ag} 4 \mathrm{~d}$ and $\mathrm{O} 2 \mathrm{p}$ states, the effective mass of the defect band is small which leads to improvement in the transport properties. $^{22}$ Furthermore, it has also been possible to synthesize dilute magnetic semiconductors with a ferromagnetic behaviour by silver doping in the $\mathrm{ZnO}$ wurtzite structure that can be utilised in spintronic applications. ${ }^{23}$ Therefore, heterostructures comprising of semiconducting $\mathrm{ZnO}$ and plasmonic $\mathrm{Ag}$ with well-defined morphologies have attracted significant interest owing to their potential for providing new opportunities for optimising, tuning and/or enhancing the optical properties, electronic structure, electrical characteristics, and light-induced charge separation effects. ${ }^{24-26}$ These plasmonic/dielectric heterostructures have gained considerable attention for studying their absorption spectral features, ${ }^{27}$ photoluminescence, ${ }^{28}$ Raman scattering, ${ }^{29}$ second harmonic generation, ${ }^{30}$ electrical conductivity, ${ }^{31,32}$ and photocatalytic ${ }^{33}$ and biological activity. ${ }^{34,35}$ There have been extensive studies on the synthesis, characterization and applications of heteronanostructures; however, hardly any attention has been paid to the investigation of these aspects in microstructures.

In this article, we elucidate the modification in spectral characteristics of the $\mathrm{Ag} / \mathrm{ZnO}$ heteromicrostructures upon injection of plasmonic hot electrons at the metal-semiconductor interface. Heterostructures with diverse morphologies have been synthesised by in situ hydrolysis of mixed metal ions of variable compositions in water/o-xylene binary solvent mixtures under alkaline conditions. A salient feature of physical significance is that a small variation in the experimental conditions creates diversity in the morphology and therefore, spectral characteristics of the $\mathrm{Ag} / \mathrm{ZnO}$ heterostructures. Thus, silver/zinc oxide has been adopted as the typical model metal-semiconductor system to understand the light-induced photophysical processes under variable microenvironmental conditions.

\section{Experimental}

\section{Reagents and instruments}

All reagents used were of analytical reagent grade. Zinc acetate dihydrate $\left[\mathrm{Zn}\left(\mathrm{OOCCH}_{3}\right)_{2} \cdot 2 \mathrm{H}_{2} \mathrm{O}\right]$, silver nitrate $\left(\mathrm{AgNO}_{3}\right)$ and potassium hydroxide $(\mathrm{KOH})$ were purchased from SigmaAldrich and were used as received. Methanol and $o$-xylene were purchased from Sisco Research Laboratories, India, and were 
used without further purification. Double distilled water was used during the course of this investigation.

Absorption spectra were measured in a PerkinElmer Lambda750 spectrometer using $1 \mathrm{~cm}$ quartz cuvette. Diffuse reflectance spectra were measured using the same instrument taking the solid sample in a reflectance port in a $100 \mathrm{~mm}$ integrating sphere. Accessories and baseline were corrected by using a reference reflectance port in the integrated sphere. Photoluminescence spectra were recorded on a PerkinElmer LS-45 spectrofluorimeter equipped with a $9.9 \mathrm{~W}$ xenon flash lamp and a photomultiplier tube with the S-20 spectral response. The spectrofluorometer was linked to a personal computer and the FL Winlab software package was utilized for data collection and processing. Scanning electron micrographs were recorded by using a JEOL JSM6360 instrument equipped with a field emission cathode with a lateral resolution of approximately $3 \mathrm{~nm}$ and acceleration voltage of $3 \mathrm{kV}$ after sputtering the sample on a silicon wafer with carbon (approx. $6 \mathrm{~nm}$ ). Thin films were prepared by drop-coating from the methanolic solutions of the respective samples onto silicon wafers. Energy dispersive X-ray (EDX) analysis was performed using the Bruker's QUANTAX microanalysis system. Fluorescence microscopy images of the membrane were recorded on a Zeiss fluorescence microscope (excitation: $325 \mathrm{~nm}$, detection: $540 \mathrm{~nm}$ ). Transmission electron microscopy (TEM) was carried out on a JEOL JEM-2100 microscope with a magnification of $200 \mathrm{kV}$. Samples were prepared by placing a drop of solution on a carbon-coated copper grid and dried overnight under vacuum. High resolution transmission electron micrograph (HRTEM) and selected area electron diffraction (SAED) patterns were obtained using the same instrument. Fourier transform infrared spectra were recorded in the form of pressed $\mathrm{KBr}$ pellets in the range of $400-4000 \mathrm{~cm}^{-1}$ on a ShimadzuFTIR Prestige-21 spectrophotometer. Cyclic voltammetry measurements were performed on a CHI-660C electrochemical workstation. Data have been collected using Pt electrodes as working as well as counter electrodes and an $\mathrm{Ag} / \mathrm{AgCl}$ electrode (in $0.1 \mathrm{M} \mathrm{KCl}$ solution) as the reference electrode at a temperature of $298 \mathrm{~K}$. Powder X-ray diffraction patterns were obtained using a Bruker AXS D8 ADVANCE X-ray diffractometer with CuK $\alpha$ radiation $(\lambda=1.4506 \AA)$; data were collected at a scan rate of $0.5^{\circ} \mathrm{min}^{-1}$ in the range of $10^{\circ}-80^{\circ}$. X-ray photoelectron spectroscopy measurements were performed using a PHI-Quantum-2000 X-ray photoelectron spectrometer with $\mathrm{AlK}_{\alpha}$ radiation. Raman measurements were performed using a micro-Raman set-up consisting of a spectrometer (LabRAM HR JovinYvon) and a Peltier-cooled CCD detector. An air-cooled argon-ion laser $\left(\mathrm{Ar}^{+}\right)$with a wavelength of $488 \mathrm{~nm}(2.55 \mathrm{eV})$ was used as an excitation light source, and a $10 \times$ objective with a numerical aperture of 0.9 was used to focus the laser and to collect the scattered light from the sample. The COMSOL Multiphysics software package was used for the finite element method.

\section{Results and discussion}

In this experiment, silver/zinc oxide microstructures with different interesting morphologies have been synthesised by in situ hydrolysis of mixed metal ions of variable compositions in a water/o-xylene binary solvent mixture under alkaline conditions as has been described in detail in ESI1. $\dagger$ The manipulation of the concentration of the silver precursor has been found to be an effective strategy for tailoring the morphology of the plasmonic/semiconductor heterostructures. In this synthetic strategy, Ag seeds formed in situ catalyse the subsequent growth of $\mathrm{ZnO}$ on the surface of metallic particles; thus, the simultaneous hydrolysis of the precursor salts via a thermal method induces microscale co-organisation of semiconducting $\mathrm{ZnO}$ and plasmonic $\mathrm{Ag}$ particles and provides a rational approach for the formation of metal/semiconductor microstructures. Thus, five different sets of the assemblies were prepared with molar concentration ratios of zinc and silver precursor salts as 1:0.010, 1:0.013, 1:0.020, 1:0.040 and $1: 0.100$, designated as sets A, B, C, D and E, respectively as has been presented in ESI2. $\dagger$ For the purpose of comparison, studies have been included for ZnO quantum dots (QDs) $(3 \pm 0.5 \mathrm{~nm})$ synthesised using the same synthetic methodology except that no silver precursor was added in the reaction mixture. ${ }^{36}$ The resultant assemblies so obtained have been characterised by diffuse reflectance spectroscopy (DRS), Fourier transform infrared (FTIR) spectroscopy, cyclic voltammetry (CV), Raman spectroscopy, selected area electron diffraction (SAED), X-ray diffraction (XRD) and energy dispersive X-ray (EDX) analysis, X-ray photoelectron spectroscopy (XPS), fluorescence microscopy, transmission electron microscopy (TEM), high resolution transmission electron microscopy (HRTEM), and scanning electron microscopy (SEM).

The morphology, composition and crystallinity of the particles have been characterised by TEM, SEM, fluorescence microscopic imaging, EDX analysis and SAED. Typical scanning electron microscopy images, fluorescence microscopy images and energy dispersive X-ray patterns of the $\mathrm{Ag} / \mathrm{ZnO}$ microstructures are shown in Fig. 1. Panels (a-e) exhibit low resolution SEM images of the as-prepared heterostructures synthesised in the presence of different concentrations of the silver precursor. It can be clearly observed that variation of the silver precursor concentration in the reaction mixture in the presence of water/ $o$-xylene immiscible liquid pairs under boiling conditions (heating at $60{ }^{\circ} \mathrm{C}$ ) gives rise to morphological diversity with specific structural features. Insets in the panels (a-e) show the high resolution scanning electron micrographs of the respective heterostructures. A closer inspection of the morphology shows that the architectures become more and more complex with increase in the silver precursor concentration. More precisely, it is noted that with increase in the silver precursor concentration, a variation of the shape from roughened microrods to cuboidalshaped microrods to urchin-like to multipod-like to flower-like microstructures becomes apparent. From these structural investigations, it is apparent that the concentration of the silver precursor plays a critical role in the evolution of diverse microstructures due to the change in the concentration gradient and solubility of the solutes in the solvent pair. ${ }^{37}$ Zinc acetate and silver nitrate are soluble in water $\left(430 \mathrm{~g} \mathrm{~L}^{-1}\right.$ and $2220 \mathrm{~g} \mathrm{~L}^{-1}$ at $20^{\circ} \mathrm{C}$, respectively); however, there is no report of 

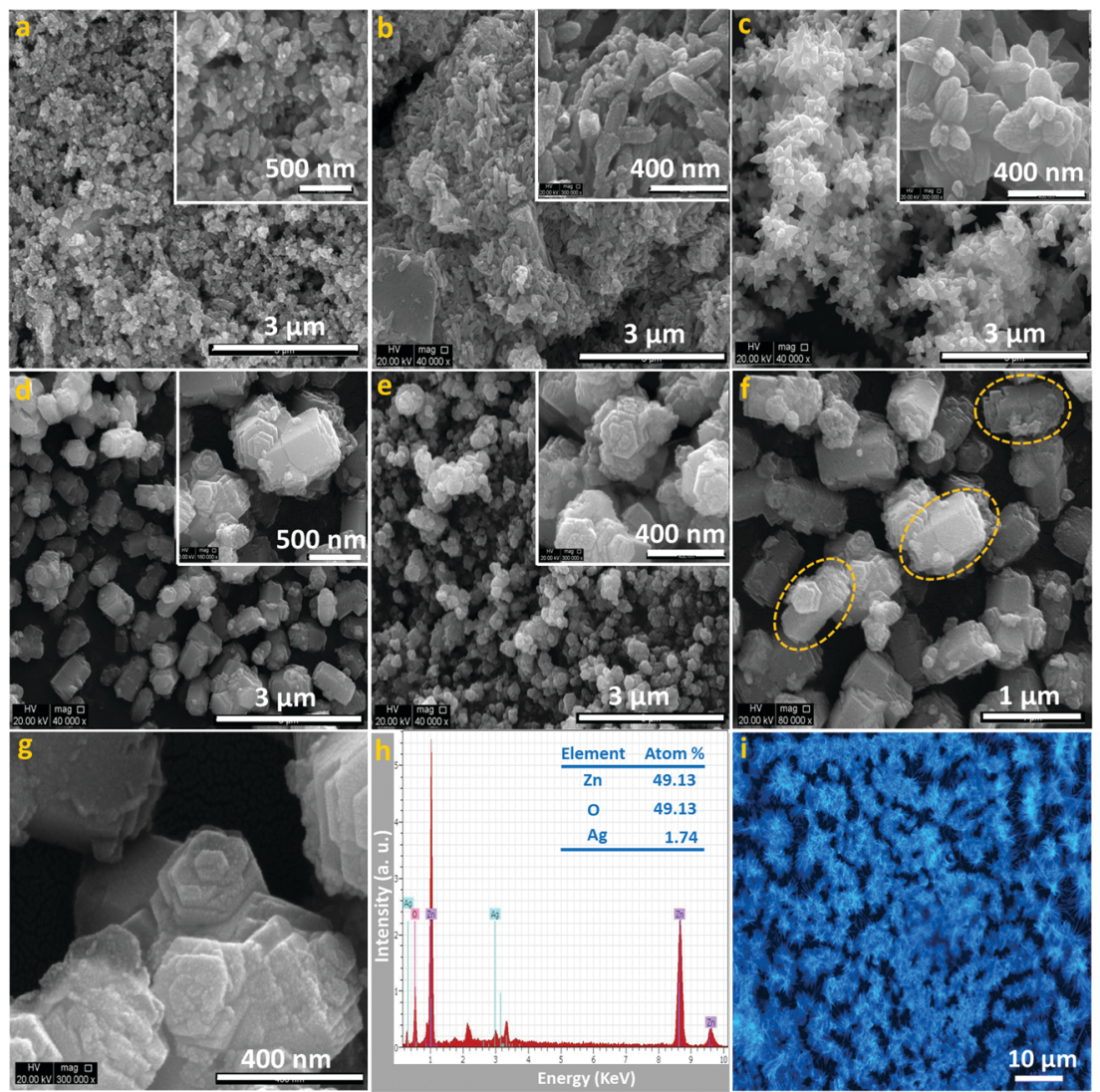

Fig. 1 (a-e) Scanning electron micrographs of Ag/ZnO heterostructures with $\mathrm{ZnO}: \mathrm{Ag}=1.0: 0.01,1.0: 0.013,1.0: 0.02,1.0: 0.04$ and $1.0: 0.1$, respectively; (f) high resolution SEM image of the architectures (set D) indicating the assemblies the smaller particles, (g) high resolution SEM image of the architectures (set E) showing the tight binding of the particles, (h) energy dispersive X-ray spectrum (set C) and (i) fluorescence microscopic image of the architectures (set C) under bright field.

solubility of zinc acetate or silver nitrate in $o$-xylene. Although the polarity of $o$-xylene is negligible, the solvent renders a unique environment (the miscibility of $o$-xylene in water is $0.02 \%$ at $20{ }^{\circ} \mathrm{C}$ ) in the formation of the architectures. However, it can be assumed that $o$-xylene has two roles: firstly, it may decrease the solubility of zinc acetate as well as silver nitrate because of the lyophobic nature and secondly, due to the difference in viscosity of the solvents (water: $1.0 \mathrm{cP}$ and $o$-xylene: $0.81 \mathrm{cP}$ at $20^{\circ} \mathrm{C}$ ), the migration behavior in the organic solvent is different from that in water which offers further possibilities to control the mobility of the ions. Therefore, during the reaction, changing the ratio of the ions controls the morphology of the heterostructures. From the representative high magnification SEM image (panel f) of the heterostructures (set D), the observation of the "broken off" $\mathrm{ZnO}$ arms indicates the assembly of the smaller particles in harnessing the microstructures. ${ }^{38}$ The high resolution SEM image (panel g) of the heterostructures (set E) exhibits that the particles are tightly packed in the microstructures. The compositional profile obtained by the representative energy dispersive X-ray spectrum (panel h) shows that the heterostructures (set C) are composed of $\mathrm{Zn}, \mathrm{O}$ and $\mathrm{Ag}$ elements with the atomic ratio of $1: 1$ : 0.035 , which confirms the deposition of silver species and the absence of other impurities in the as-prepared heterostructures. ${ }^{37}$ The representative fluorescence microscopy image (in the bright field) of the resultant heterostructures (set C) is shown in panel i; the heterostructures so formed were collected in a micropipette and suspended in water in the cavity of a glass slide. The observation of structurally intact assemblies exhibits the crystallisation of fluorescent $\mathrm{ZnO}$ particles into microstructures. ${ }^{39}$

The structural morphology of the as-synthesised heterostructures has been further confirmed by TEM, HRTEM and selected area electron diffraction patterns as shown in Fig. 2. Panel a shows the low-magnification TEM image taken from the edge of the as-prepared $\mathrm{Ag} / \mathrm{ZnO}$ heterostructures (set $\mathrm{E}$ ) which reveals that the structure is comprised of both metallic and semiconductor components. The substantially higher mass contrast sensitivity in the image causes the silver nanoparticles to appear much brighter than the surrounding $\mathrm{ZnO}$ architecture. The transmission electron micrographs were measured for all sets of particles; it was observed that the size of the silver particles lies in the range of $16 \pm 4 \mathrm{~nm}$ and does not change considerably in different sets of the hererostructures. Panel $b$ shows the HRTEM image of the Ag/ZnO heterostructures (set E) that exhibits the lattice fringes as $0.25 \mathrm{~nm}$ belonging to the (111) plane of fcc Ag and $0.26 \mathrm{~nm}$ of the (002) plane of wurtzitetype hexagonal $\mathrm{ZnO}$ at the edge portion, confirming the crystalline nature of $\mathrm{Ag}$ and $\mathrm{ZnO}$ components. ${ }^{40}$ The corresponding SAED pattern (panel c) of Ag/ZnO heterostructures (set E) exhibits 


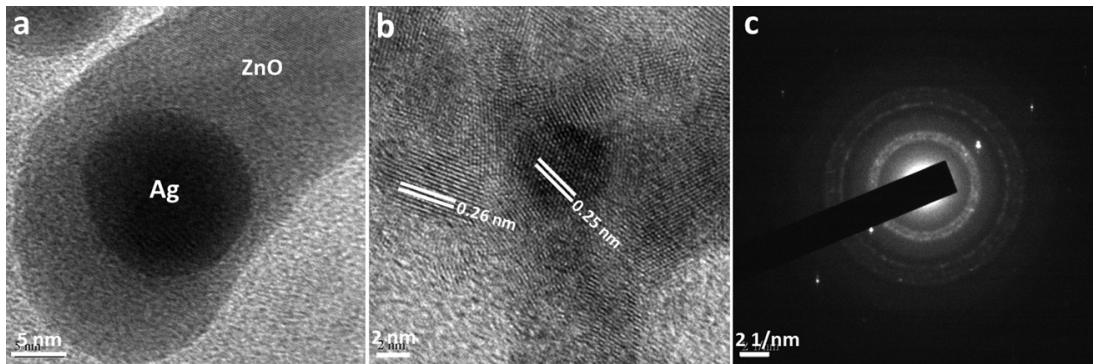

Fig. 2 (a) Transmission electron micrograph, (b) high resolution transmission electron micrograph and (c) selected area electron diffraction pattern, corresponding to $\mathrm{Ag} / \mathrm{ZnO}$ heterostructures (set $\mathrm{E}$ ).

polycrystalline-like diffraction which is consistent with the reflections (100), (002), (101), (102), (110) corresponding to the hexagonal wurtzite phase of ZnO particles along with the (111), (200) and (220) diffraction lines of Ag which reveals crystallographic orientations for both $\mathrm{ZnO}$ and $\mathrm{Ag}$ in the heterostructures. ${ }^{40}$

Since microscopic analysis shows that the as-prepared microstructures are not smooth, the optical absorption of the samples has been measured by diffuse reflectance rather than by normal reflectance. Fig. 3 shows the diffuse reflectance spectra of the five different sets of $\mathrm{Ag} / \mathrm{ZnO}$ microstructures in the solid state. The strong opalescence of the microstructures shows two maxima: one is in the range of $300-350 \mathrm{~nm}$, which is characteristic of the $\mathrm{ZnO}$ direct band gap transition ${ }^{41}$ and other is near $400 \mathrm{~nm}$ which arises due to the localised surface plasmon absorption band of the silver nanoparticles. ${ }^{18}$ Under plasmon resonance conditions, an evanescent electric field is created on the surface of the silver nanoparticles that penetrates into the surrounding $\mathrm{ZnO}$ and augments the electric field of the illuminating light. ${ }^{25}$ It is seen that the positions of the maxima of both $\mathrm{ZnO}$ and $\mathrm{Ag}$ are different in the various sets, which indicates that the spatial distribution of the electrons is no more homogeneous and is modified at the interface. This is because the incorporation of silver increases the density of

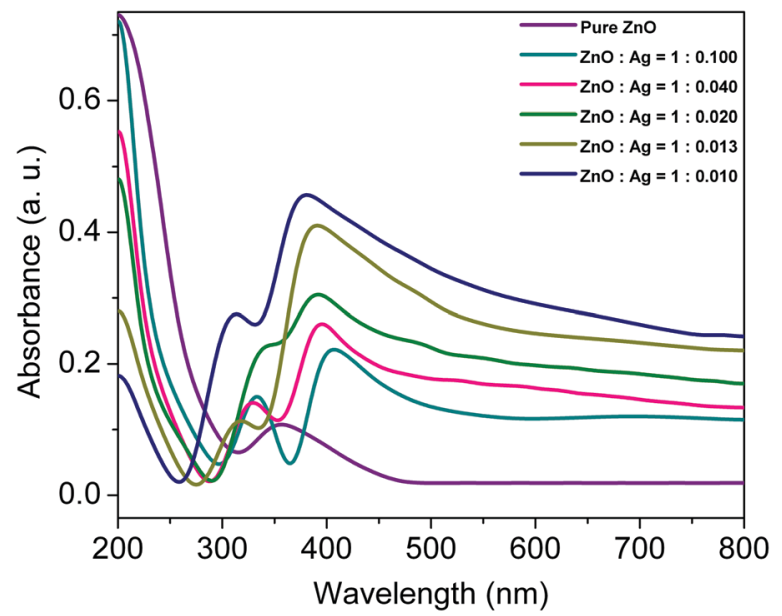

Fig. 3 Diffuse reflectance spectra of pure $\mathrm{ZnO}$ and different sets of $\mathrm{Ag} /$ $\mathrm{ZnO}$ microstructures. states impinging the photoexcited electrons of the silver nanostructures near the Fermi level of $\mathrm{ZnO}$, thereby leading to the modification of the band structure at the $\mathrm{Ag} / \mathrm{ZnO}$ interface. ${ }^{42}$ The efficiency of manipulation of the electromagnetic radiation, critically depends on the concentration of the metallic particles as well as the integration of plasmonic and semiconductor components within the microstructures. ${ }^{43}$ However, a regular change in the reflectance spectra was not seen with the increase in the silver nitrate concentration which can be ascribed to the interplay of different positional and orientational factors of the individual components within the $\mathrm{Ag} / \mathrm{ZnO}$ microstructures.

The engineering of band gaps is common practice for exploiting semiconductors for desired physical properties. ${ }^{44}$ The electronic structure of semiconductors, such as, $\mathrm{ZnO}$ is characterised by a valence band, which is filled with electrons, and an empty conduction band. In metal/semiconductor microstructures, photoinduced charge carriers are trapped by the noble metal particles and become capable of promoting the interfacial charge-transfer processes. ${ }^{45}$ The optical band gap of the pure $\mathrm{ZnO}$ and the microstructures can be estimated using Tauc's equation, $(\alpha h \nu)^{1 / n}=A^{1 / n}\left(h \nu-E_{\mathrm{g}}\right)$, where, $\alpha$ is the absorption coefficient, $h \nu$ the photon energy, $A$ a constant and $E_{\mathrm{g}}$ represents the optical bandgap. In this equation, the value of $n=1 / 2$, for direct allowed transition. Fig. 4 shows the plot of $(\alpha h \nu)^{2}$ as a function of photon energy $(h \nu)$ of the as-synthesised pure $\mathrm{ZnO}$ and five different sets of $\mathrm{Ag} / \mathrm{ZnO}$ microstructures. Although the band gap of ZnO QDs has been calculated to be $3.92 \mathrm{eV}$, upon addition of silver for maneuvering $\mathrm{Ag} / \mathrm{ZnO}$ microstructures, the deep level emission (DLE) band becomes significant as compared to near band edge emission (NBE) band and as a consequence, the values of the band gap are reduced to $c a$. 2.48, 2.45, 2.42, 2.29 and $2.19 \mathrm{eV}$ with a decrease in the silver precursor concentration consistent with earlier observations ${ }^{46}$ A significant variation in the band gap is noted due to the incorporation of silver nanostructures in the $\mathrm{ZnO}$ matrix. The decrease in the band gap energy could be attributed to the formation of intermediate donor levels below the conduction band, with the electron requiring less energy to move from the valence to the conduction band. ${ }^{47}$ It is, therefore, apparent that manipulating and guiding photons at the microscale inside semiconductor could, sensitively, be managed with the assistance of interdigitated plasmonic metal particles. ${ }^{48}$ 


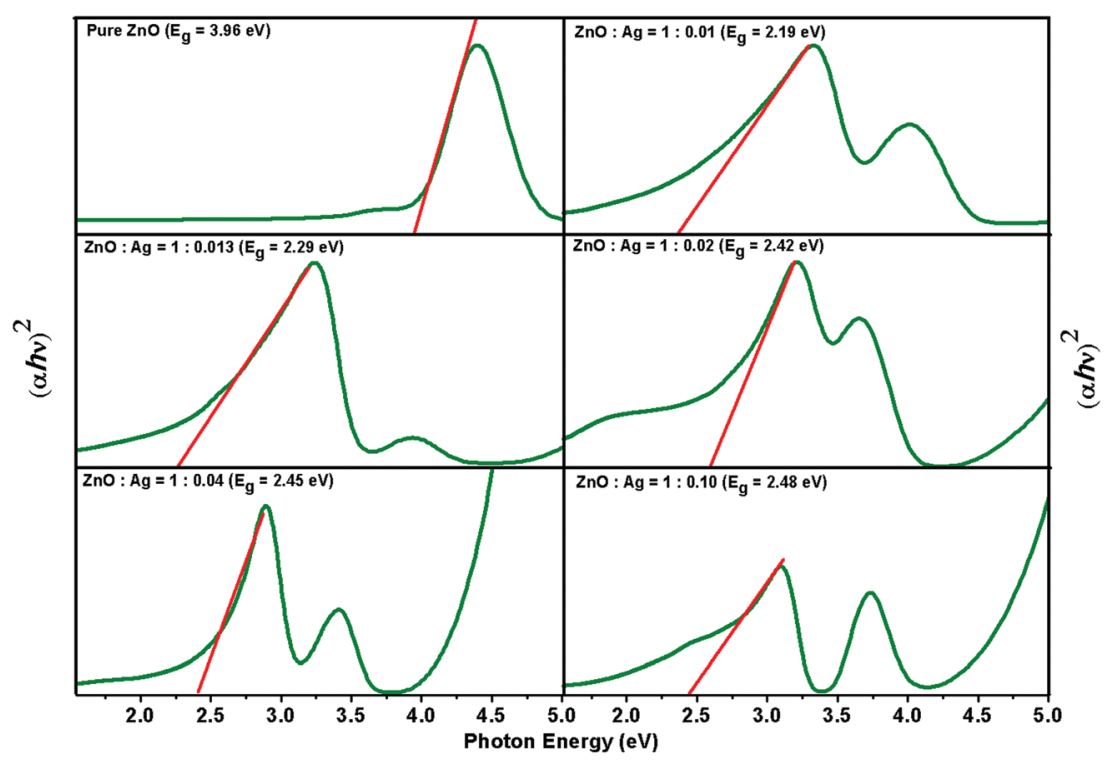

Fig. 4 Tauc plot for the estimation of the band gap energy of pure $\mathrm{ZnO}$ and different sets of $\mathrm{Ag} / \mathrm{ZnO}$ microstructures.

Fourier transform infrared spectra of the as-prepared pure $\mathrm{ZnO}$ and $\mathrm{Ag} / \mathrm{ZnO}$ microstructures (set $\mathrm{E}$ ), recorded in the range $400-4000 \mathrm{~cm}^{-1}$, are shown in Fig. 5. The FTIR spectrum of pure $\mathrm{ZnO}$ (trace a) shows a peak at $c a .453 \mathrm{~cm}^{-1}$ that can be assigned to stretching vibrations of $\mathrm{Zn}-\mathrm{O}$ bonds; the other absorption band at $1263 \mathrm{~cm}^{-1}$ corresponds to the $\mathrm{C}-\mathrm{O}$ stretching frequency due to adsorbed acetate counter ions. ${ }^{49}$ Upon addition of the silver precursor (trace b), the FTIR spectrum exhibits the appearance of two bands at $\sim 437$ and $466 \mathrm{~cm}^{-1}$ that can be assigned to the characteristic stretching modes of $\mathrm{Ag}-\mathrm{O}$ and $\mathrm{Zn}-\mathrm{O}$ bonds, respectively. ${ }^{49}$ Moreover, the presence of extra bands at 1395 and $1663 \mathrm{~cm}^{-1}$ could be attributed to the

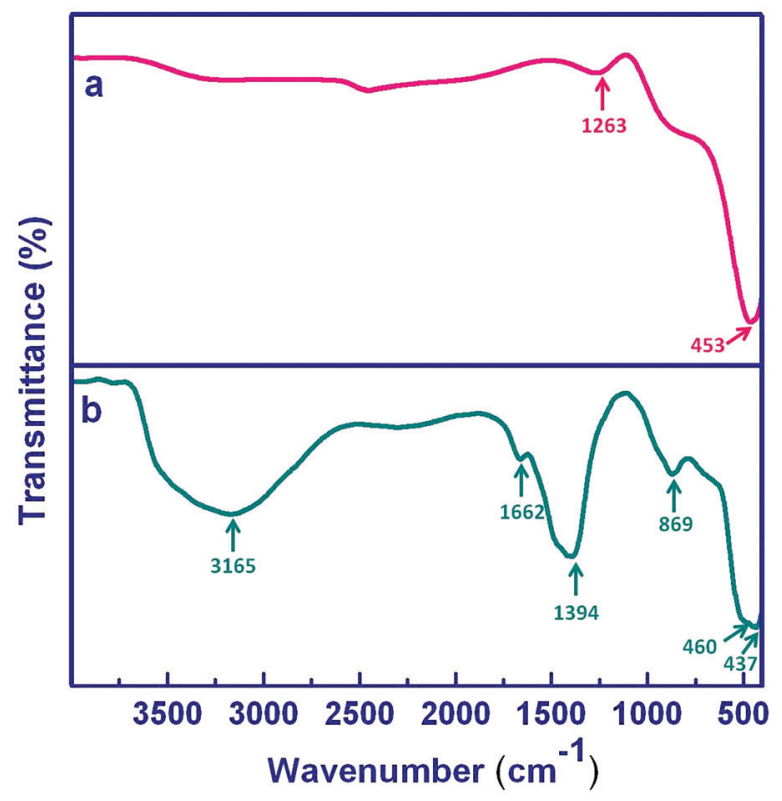

Fig. 5 Fourier transform infrared spectrum of (a) pure $\mathrm{ZnO}$ and (b) Ag/ $\mathrm{ZnO}$ microstructures (set A). asymmetric and symmetric $\mathrm{COO}^{-}$stretching vibrations of acetate chemisorption, respectively, which probably, accounts for colloid stabilisation. ${ }^{49}$ The explicit appearance of these bands in the assemblies in comparison with pure $\mathrm{ZnO}$ are possibly due to the large number of $\mathrm{ZnO}$ species present in the microstructures. The absorption bands at $c a .869$ and $3165 \mathrm{~cm}^{-1}$ arise due to the stretching modes of $\mathrm{O}-\mathrm{H}$ groups which reveals the existence of a small amount of water chemisorbed and/or physisorbed by the $\mathrm{Ag} / \mathrm{ZnO}$ architectures. ${ }^{49}$

Powder X-ray diffraction patterns of the as-prepared pure $\mathrm{ZnO}$ and five different sets of $\mathrm{Ag} / \mathrm{ZnO}$ microstructures are shown in Fig. 6. All the diffraction peaks of pure $\mathrm{ZnO}$ could be indexed as the pure hexagonal phase of $\mathrm{Zn}$ with a space group of $C_{6 \mathrm{v}}^{4}$ and cell constants $a=3.25 \AA$, and $c=5.21 \AA$ (JCPDS: 76-0704), which suggests that the product comprises $\mathrm{ZnO}$ with the wurtzite structure. ${ }^{50}$ Upon addition of the silver precursor and subsequent hydrolysis in harnessing $\mathrm{Ag} / \mathrm{ZnO}$ microstructures, in addition to the incipient peaks, additional diffraction peaks at $c a .38 .1^{\circ}, 44.3^{\circ}$ and $64.47^{\circ}$ (JCPDS 87-0597) corresponding to the diffraction lines of (111), (200) and (220), respectively can be indexed to the face-centred cubic structure of Ag. ${ }^{50}$ The appearance of silver peaks becomes prominent with increase in silver precursor concentration; the low intensity of the silver peaks could be attributed to an insufficient concentration of silver in the heterostructures.

The mechanism of the emergence of morphological diversity upon increase in the silver precursor concentration has been confirmed by multiple control experiments. It has, now, been established that formation of such architectures occurs through the spatial arrangement governed by different intramolecular or intraparticulate interactions, such as electrostatic interaction, cross-linking agents, hydrogen bonding, charge transfer interaction, van der Waals forces, dipole-dipole interaction etc. amongst the individual building blocks. ${ }^{51}$ The evolution into microstructures was not seen in the absence of 


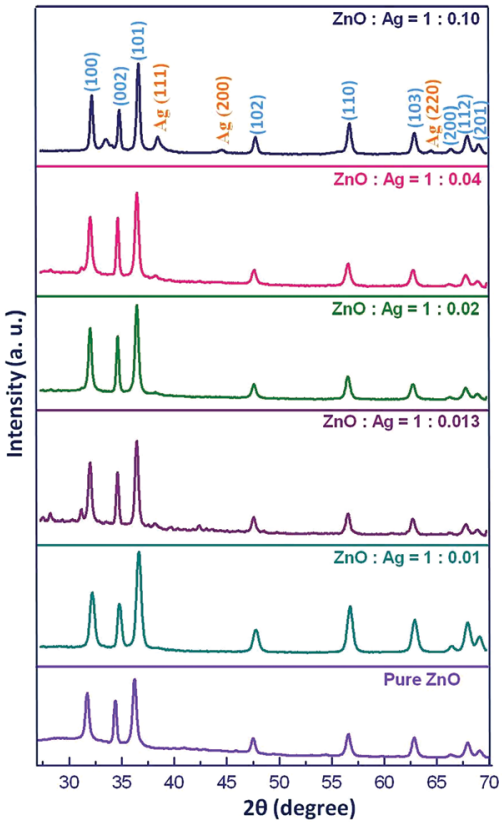

Fig. $6 \mathrm{X}$-ray diffraction patterns of pure $\mathrm{ZnO}$ and different sets of $\mathrm{Ag} / \mathrm{ZnO}$ microstructures.

silver precursor; therefore, in this synthesis strategy, the in situ generated $\mathrm{Ag}$ seeds govern the subsequent nucleation of $\mathrm{ZnO}$, which has a great influence on the final morphologies of $\mathrm{ZnO}$ mediated by silver ion concentration in the reaction mixture. The formation of such specific architectures is also not observed in the absence of $o$-xylene in the solvent medium; thus, it is apparent that the observed morphology is the result of specific interactions governed by the interfacial tension at the water-organic interface that enable effective interparticle interactions in harnessing the microstructures. ${ }^{52}$ Therefore, it could be conceived that the smaller particulates in solution with the well matched crystal lattice and active surface are prone to integrate together through the oriented attachment, driven by the increase of free energy and lattice free energy. ${ }^{53}$ The mechanism of evolution of $\mathrm{Ag} / \mathrm{ZnO}$ microstructures (set E) through kinetic studies based on absorption, Fourier transform infrared and X-ray diffraction spectroscopy is enunciated in ESI3. $\dagger$

X-ray photoelectron spectroscopy analysis has been carried out for further confirmation of the purity and the composition of the $\mathrm{Ag} / \mathrm{ZnO}$ microstructures (set C). The XPS spectra demonstrate the presence of $\mathrm{Zn} 2 \mathrm{p}, \mathrm{O} 1 \mathrm{~s}$ and $\mathrm{Ag} 3 \mathrm{~d}$ as shown in Fig. 7. The Zn 2p (panel a) exhibits two significant binding energy peaks at $1043.2 \mathrm{eV}$ (1044.4 eV in the bulk) and $1020.0 \mathrm{eV}$ (1021.65 eV in the bulk) that can be attributed to $2 \mathrm{p}_{1 / 2}$ and $2 \mathrm{p}_{3 / 2}$, respectively. The splitting distance $23.2 \mathrm{eV}$ between the two peaks indicates that the $\mathrm{Zn}$ element existed in the form of $\mathrm{Zn}^{2+}{ }^{24}$ The $\mathrm{O}$ 1s peak (panel b) shows the position of binding energy at $532.6 \mathrm{eV}$ associated with the lattice oxygen in the semiconductor matrix. Similarly, the Ag 3d (panel c) binding energy peaks are located at $372.4(373.4 \mathrm{eV}$ in bulk) and $366.4 \mathrm{eV}$ (368.22 eV in the bulk) corresponding to $3 \mathrm{~d}_{3 / 2}$ and $3 \mathrm{~d}_{5 / 2}$, respectively. The significant shift in binding energies of $\mathrm{Zn} 2 \mathrm{p}_{3 / 2}$ and $\mathrm{Ag} 3 \mathrm{~d}_{5 / 2}$ from the corresponding values of the bulk can be attributed to electron transfer from metallic $\mathrm{Ag}$ to $\mathrm{ZnO}$ crystals. ${ }^{55}$ When the metallic and semiconductor components come in contact with each other, the position of the corresponding Fermi level is equilibrated which results in excess of free electrons on the new Fermi level of the metallic components. As a consequence, the free electrons can tunnel into the vacant conduction band of the $\mathrm{ZnO}$, resulting in a higher valence state of $\mathrm{Ag}$ components. ${ }^{56}$

Raman spectroscopy is the most effective technique to achieve sensitive detection of optical phonon modes and therefore to characterise related vibrational properties. Fig. 8 presents the room temperature Raman spectra of pure $\mathrm{ZnO}$ and $\mathrm{Ag} / \mathrm{ZnO}$ microstructures in the $50-2000 \mathrm{~cm}^{-1}$ spectral range; in the present experiment, the samples were excited by the $488 \mathrm{~nm}$ line $(2.55 \mathrm{eV})$ of an $\mathrm{Ar}^{+} \cdot$ laser. It is seen that the spectrum of $\mathrm{ZnO}$ is very weak; however, the intensity of the peaks increases with increase in the silver precursor concentration; surface enhanced Raman scattering is, therefore, observed in the spectrum of $\mathrm{Ag} / \mathrm{ZnO}$ microstructures. According to group theory, the structure of $\mathrm{ZnO}$ belongs to the $C_{6 \mathrm{v}}^{4}$ symmetry group having two formula units per primitive cell with all the atoms occupying the $C_{3 \mathrm{v}}$ sites, which predicts eight sets of zone centre optical phonons: two $A_{1}$, two $E_{1}$, two $E_{2}$ and two $B_{1}$ modes. Among these, $A_{1}$ and $E_{1}$ modes are polar and split into transverse $\left(A_{1} T\right.$ and $\left.\mathrm{E}_{1} \mathrm{~T}\right)$ and longitudinal $\left(\mathrm{A}_{1} \mathrm{~L}\right.$ and $\left.\mathrm{E}_{1} \mathrm{~L}\right)$ phonons, all being Raman and infrared active; $\mathrm{E}_{2}$ modes are non-polar consisting of two modes of low- and high-frequency $\left(\mathrm{E}_{2} \mathrm{~L}\right.$ and $\left.\mathrm{E}_{2} \mathrm{H}\right)$ phonons and are only Raman active, and $\mathrm{B}_{1}$ modes are infrared and Raman inactive (silent modes) ${ }^{29}$ The low frequency $\mathrm{E}_{2}$ mode is observed
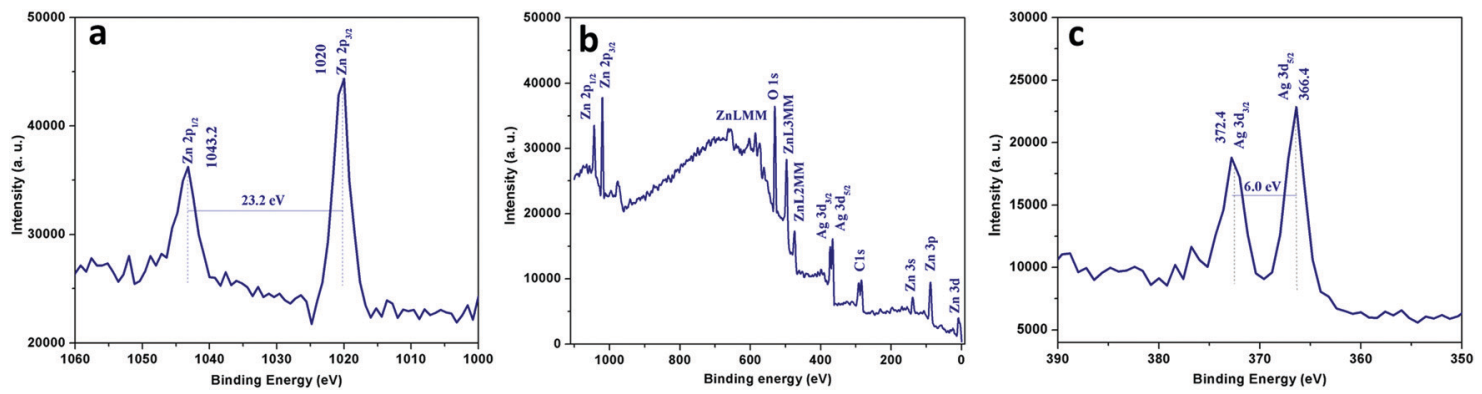

Fig. 7 X-ray photoelectron spectra corresponding to (a) Zn 2p, (b) O 1s and (c) Ag 3d of the representative Ag/ZnO microstructures. 


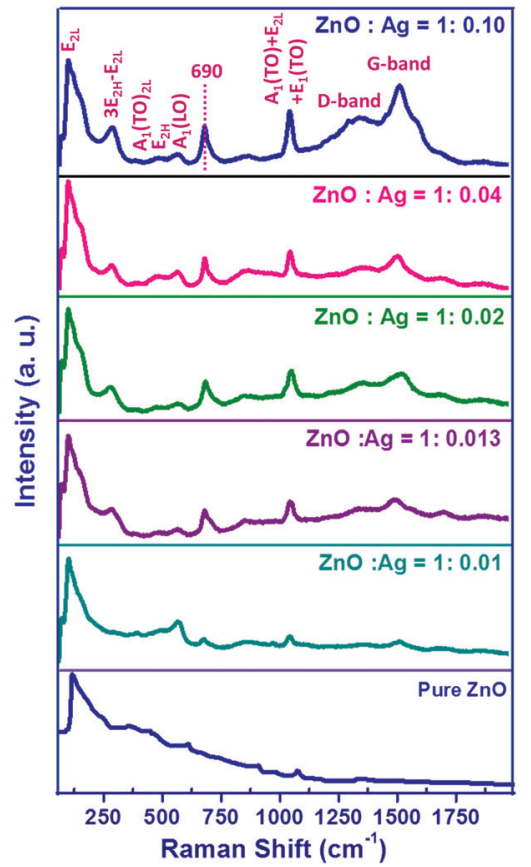

Fig. 8 Raman spectra of pure $\mathrm{ZnO}$ and different sets of $\mathrm{Ag} / \mathrm{ZnO}$ microstructures

at $100 \mathrm{~cm}^{-1}$ which is associated with the vibration of the $\mathrm{Zn}$ sub-lattice while the high frequency $\mathrm{E}_{2}$ mode is related to the vibration of only oxygen atoms and is considered as the Raman fingerprint of the wurtzite $\mathrm{ZnO}$ phase. ${ }^{29}$ The $\mathrm{A}_{1}(\mathrm{TO})$ and $\mathrm{A}_{1}(\mathrm{LO})$ polar branches appeared at about $380 \mathrm{~cm}^{-1}$ and $570 \mathrm{~cm}^{-1}$, respectively; the $\mathrm{A}_{1}(\mathrm{LO})$ phonon mode is commonly assigned to the oxygen vacancies, zinc interstitials, or defect complexes containing oxygen vacancies and zinc interstitials in $\mathrm{ZnO}^{29}$ Other two Raman peaks located at about 331 and $1050 \mathrm{~cm}^{-1}$ can be assigned to the $3 \mathrm{E}_{2 \mathrm{H}^{-}} \mathrm{E}_{2 \mathrm{~L}}$ and $\mathrm{A}_{1}(\mathrm{TO})+\mathrm{E}_{2 \mathrm{~L}}+\mathrm{E}_{1}(\mathrm{TO})$ multiphonon scattering modes, respectively. ${ }^{29}$ The Raman peak appeared at about $690 \mathrm{~cm}^{-1}$ has also been reported for codoped $\mathrm{ZnO}$; however, the origin of this peak is not yet clear. ${ }^{57}$ Nonetheless, the appearance of two new peaks at 1330 and $1581 \mathrm{~cm}^{-1}$ can be attributed to the D-band and G-band of acetate counter ions, respectively, responsible for the stabilisation of the microstructures. ${ }^{58}$ The appearance of these bands due to acetate counter ions at the higher silver concentration is in conformity with the infrared spectra as discussed earlier. The enhancement of the intensity of the Raman signal with increase in the silver precursor concentration can mainly ascribed to the interaction between the metallic and semiconductor components in the microstructures. ${ }^{59}$

Cyclic voltammograms of five different sets of $\mathrm{Ag} / \mathrm{ZnO}$ microstructures $(0.01 \mathrm{M})$ in $0.1 \mathrm{M} \mathrm{KCl}$ solution using $\mathrm{Ag} / \mathrm{AgCl}$ as a reference electrode and Pt electrodes as working and counter electrodes are shown in Fig. 9. It has been noted that pure $\mathrm{ZnO}$ shows the current values of 24.99 and $3.67 \mu \mathrm{A}$ at the potentials of 0.45 and $0.10 \mathrm{~V}$, respectively, whereas, silver nanoparticles exhibit no peak potentials in the pure state (not shown). From the spectra, it is seen that the microstructures do not possess any anodic peak potential which has been seen for pure $\mathrm{ZnO}$ rather a peak at $E_{\mathrm{pc}} \sim-0.7 \mathrm{~V}$ is observed with varying amount of current for different sets of $\mathrm{Ag} / \mathrm{ZnO}$ microstructures. It is also noted that the amount of current is increasing (4.901, $6.557,6.584,8.335$ and $10.385 \mu \mathrm{A}$ for the sets of A-E, respectively) with the increasing ratio of silver in the microstructures. These results reveal that a higher number of the electrons becomes transferred from $\mathrm{Ag}$ to $\mathrm{ZnO}$ with increasing the ratio of silver in the heterostructures. ${ }^{49}$ Moreover, the differences in the flow of current are related to the differences in the extent of electron transfer at the interface of the electrodes that reflects the difference in charge densities of different sets of the microstructures. ${ }^{41}$

Due to the present state-of-the art advances in the synthesis and characterisation techniques, it has been established that plasmonic metal/semiconductor microstructures are materials of immense interest due to complementarity in the optical properties of the long-lived excitons in semiconductors and the localised electromagnetic modes in metallic nanoparticles. ${ }^{60}$ Therefore, the investigation of structure-luminescence relationship is quite fascinating in shape-selective metal/semiconductor microstructures as has been presented in Fig. 10. Panel A displays the normalised photoluminescence spectra of pure $\mathrm{ZnO}$ and $\mathrm{Ag} /$ $\mathrm{ZnO}$ microstructures $\left(\lambda_{\mathrm{ex}} \sim 325 \mathrm{~nm}\right)$ in the solid state. It is seen that all the photoluminescence spectra mainly consist of two types of bands: the origin of UV luminescence can be attributed to the photo-stimulated creation of excitons having energies just below the $\mathrm{ZnO}$ band edge and their subsequent recombination (near the band edge emission band) and the other peak in the visible region may arise from the recombination of photogenerated holes with the electrons in singly occupied oxygen vacancies (deep level emission band) ${ }^{14}$ It is seen that pure $\mathrm{ZnO}$ and $\mathrm{Ag} / \mathrm{ZnO}$ microstructures exhibit distinctly different emission profile; specifically, the concentration of the silver precursor could tune the photoluminescence properties of the microstructures. Moreover, it is seen that near-band-edge

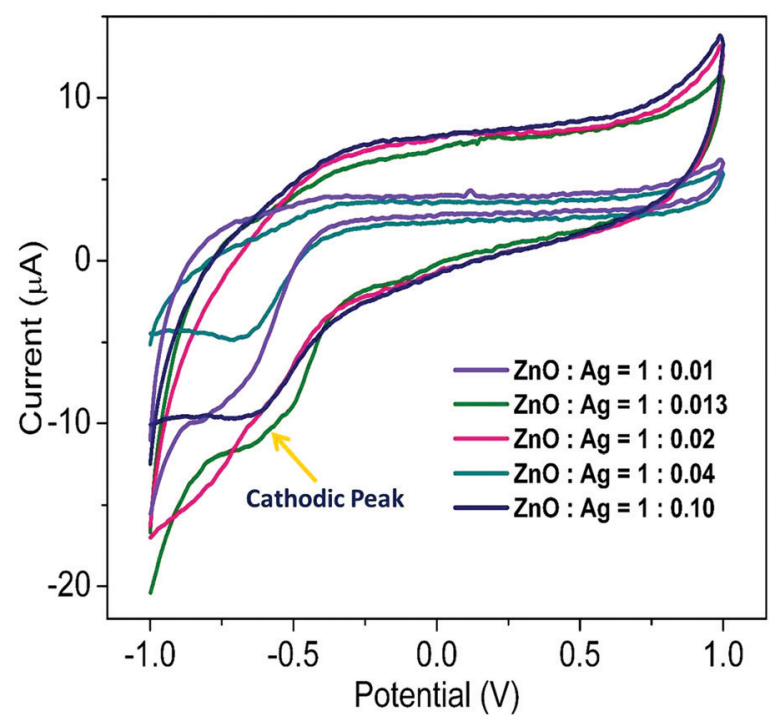

Fig. 9 Cyclic voltammograms of different sets $(0.01 \mathrm{M})$ of $\mathrm{Ag} / \mathrm{ZnO}$ microstructures. 

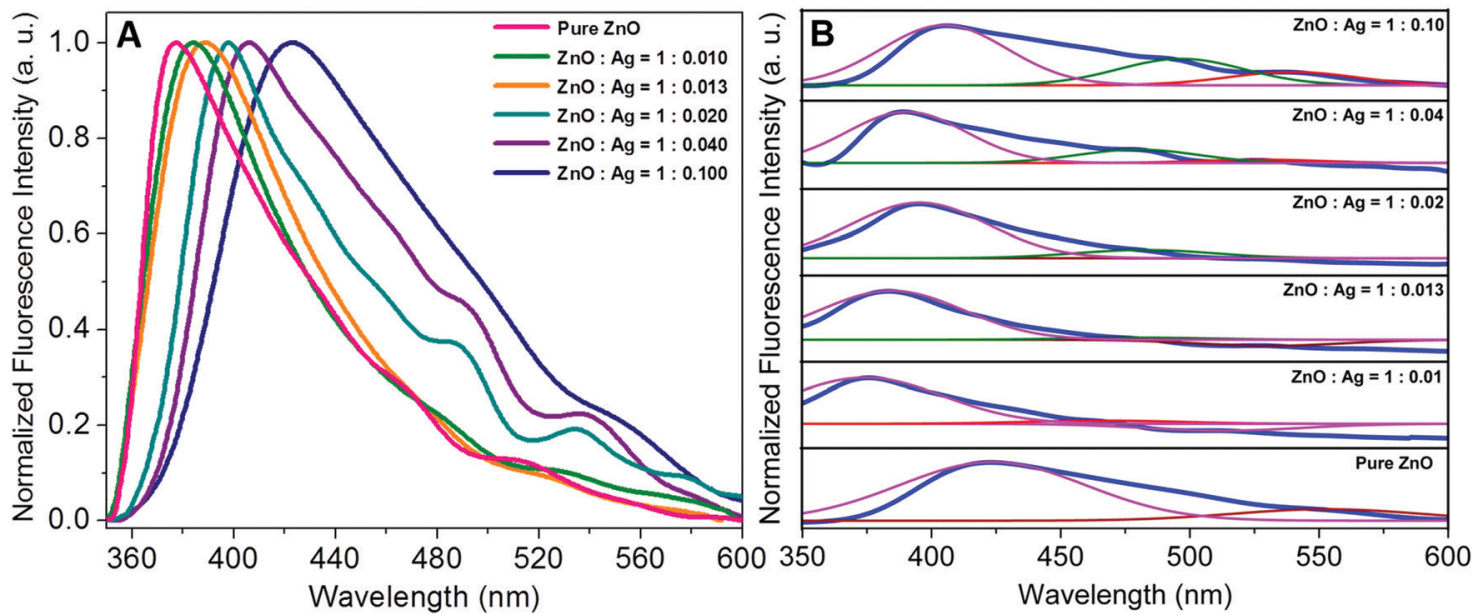

Fig. 10 (A) Photoluminescence spectra (in the solid state) and (B) deconvolution of the spectra of pure $\mathrm{ZnO}$ and different sets of $\mathrm{ZnO}-\mathrm{Ag}$ microstructures.

UV luminescence is progressively shifted to the red and appearance of new bands in the visible region with increase in silver precursor concentration. Gaussian multipeak fitting in panel B explicates the evolution of two visible emission bands in the microstructures, one at $475 \mathrm{~nm}$ and another at $525 \mathrm{~nm}$ that can be attributed to the presence of oxygen vacancy states on the surface and deep interstitial oxygen states inside the microstructures. ${ }^{50}$ The change in the photoluminescence properties of $\mathrm{ZnO}$ as a result of the assemblage implicits the strong interaction between the semiconductor and the metallic particles and points to an efficient electron transfer from $\mathrm{Ag}$ to $\mathrm{ZnO}$ components. $^{50}$

The work function of a semiconductor can be represented as $\Delta \phi=I+V_{\mathrm{bb}}-\left(E_{\mathrm{F}}-E_{\mathrm{V}}\right)=\chi+V_{\mathrm{bb}}+\left(E_{\mathrm{C}}-E_{\mathrm{F}}\right)$, where $V_{\mathrm{bb}}$ is the band bending, $\chi$ the electron affinity, $I$ the ionization energy, and $E_{\mathrm{C}}$ and $E_{\mathrm{V}}$ are the band energies of the conduction and valence bands in the bulk. ${ }^{61}$ With increase in silver deposition around the $\mathrm{ZnO}$ surface, the dipole causes a change in electron affinity or the ionization energy and space charge diffusion cause the change in the band bending potential. Considering

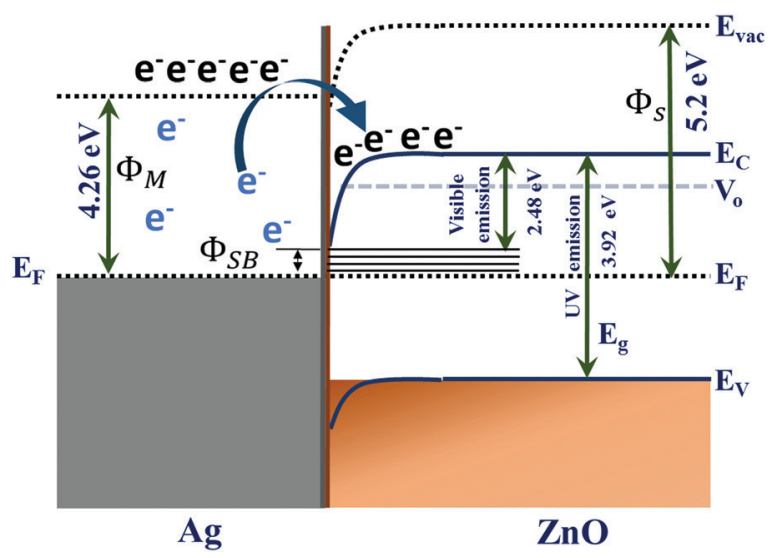

Scheme 1 Schematic presentation of the energy profile showing the electron transfer processes at the $\mathrm{Ag} / \mathrm{ZnO}$ microstructures. the change in band bending, the shift in Fermi energy level $\left(E_{\mathrm{F}}\right)$ takes the major contribution, which again depends on the dopant characteristics. When the metallic Ag and semiconducting $\mathrm{ZnO}$ species are in contact with each other, the position of the corresponding Fermi levels of both the species are equilibrated and as a consequence there is an excess of free electrons in the new Fermi level of metallic Ag components. ${ }^{62-64}$ Under such a situation, because the conduction band of the $\mathrm{ZnO}$ species is vacant, the free electrons can tunnel into the conduction band that results in a higher valence state of metallic Ag components resulting in a Schottky junction as illustrated in Scheme 1. Chernysheva et al. have shown the shift in Fermi level of $\mathrm{ZnO}$ by $\sim 0.04 \mathrm{eV}$ on the $\mathrm{Ag} / \mathrm{ZnO}$ contact. ${ }^{65}$ The work functions for $\mathrm{Ag}\left(\Phi_{\mathrm{M}}=4.26 \mathrm{eV}\right)$ and $\mathrm{ZnO}\left(\Phi_{\mathrm{S}}=5.2 \mathrm{eV}\right)$ have been adopted from the reference of Zheng et al. ${ }^{56}$ where $V_{0}$ defines the defect level and $E_{\mathrm{g}}$ the band gap of the semiconductor layer. Adsorbates modify the ionization energy and induce a change in band bending for a perfect Schottky contact (where, $\Phi_{\mathrm{SB}}$ is the Schottky barrier of the interface), and construct the interface levels, from which the transition can be shown. ${ }^{65}$ The change in the ionization energy with the increasing concentration of $\mathrm{Ag}$ on $\mathrm{ZnO}$ can be expressed as $\Delta I=\Delta \chi=e N p_{\perp}(\theta) / \varepsilon_{0}$, where, $e$ is the electronic charge, $N$ the number of dipoles and $p_{\perp}(\theta)$ the perpendicular polarisation through the adsorbate layer. Thus, with the increase in the Ag concentration around $\mathrm{ZnO}$, both of the effects lead to the decrease the difference in valence and conduction bands. Therefore, with increase in silver concentration around $\mathrm{ZnO}$, there occurs a successive red shift in photoluminescence spectra. ${ }^{29,61,65}$

We have attempted to calculate the distribution of electric field corresponding to a single silver nanoparticle in the assemblies through a finite element method. A representative schematic presentation for the calculation and the corresponding electric field distribution pattern are exhibited in Fig. 11. Plane polarised electromagnetic waves were passed through the linear arrangement assuming a concentric spherical shell around the metallic core (panel a) in the corresponding 


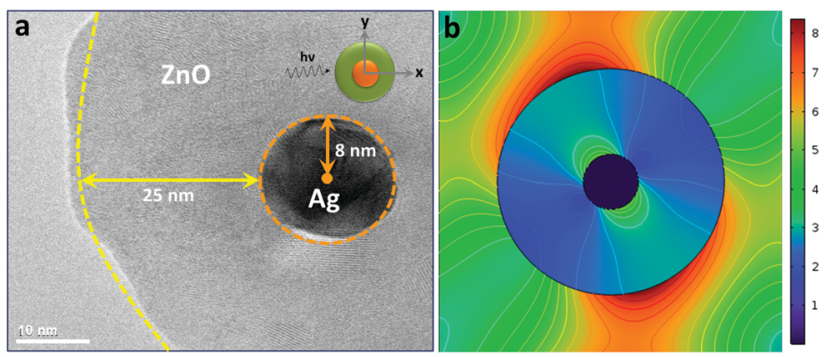

Fig. 11 (a) Representative transmission electron micrograph (set B) for parametric assignment and (b) calculation of electric field corresponding to single silver nanoparticles in $\mathrm{Ag} / \mathrm{ZnO}$ microstructures through the finite element method. Inset in panel (a) shows the polarisation direction of the incident light.

transmission electron micrograph (set B). The maximum electric field has been located along the E-field polarisation direction (y-axis) as shown in the inset. The dielectric constants for $\mathrm{ZnO}^{66}$ and $\mathrm{Ag}^{67}$ were taken as 8.5 and 2.56, respectively in this calculation. It is observed that the electric field intensity on the outer $\mathrm{ZnO}$ surface is stronger than that of the Ag (panel b). Moreover, it is noted that the enhanced local field polarises parallel to the incident polarization and concentrates on the outer surface in the ZnO shell with the maximum $\left|E^{2}\right|$ as $c a$. $8.4 \mathrm{eV}$. This can be ascribed to the presence of plasmonic field of nanostructured metallic particles to the $\mathrm{ZnO}$ shell across the interface. However, since many metal nanoparticles are embedded in the giant semiconductor matrix, the exact calculation of electric field throughout the entire microstructure warrants the modification of mathematical formulation considering the electromagnetic coupling effect in adjacent metallic nanostructures which is the subject of further investigation. ${ }^{11,68,69}$

\section{Conclusions}

In conclusion, we have elucidated the spectral properties due to interfacial interactions between plasmonic metal nanoparticles embedded in a giant semiconductor matrix. The reported synthetic strategy based on colloidal chemistry provides a facile route to the effective integration of plasmonic metal/semiconductor microstructures under a unified synthetic protocol. It has been seen that with increase in silver precursor concentration, the ease and specificity of this dual-solvent thermolytic method provides a rational approach for the synthesis of diverse microstructures. It has been resolved that the in situ nucleation and growth of the $\mathrm{Ag}$ domains followed by heterogeneous nucleation and growth of the $\mathrm{ZnO}$ component controls their spatial arrangement to induce a unique morphology with dimensions on the microscale. Spectral characterisations elucidate interfacial electron transfer at the $\mathrm{Ag} / \mathrm{ZnO}$ interface in the microstructures. Such heterogeneous microstructures present not only numerous natural interfaces for integrating multiple functions but also exhibit a substantial reduction in the band gap that may lead to the emergence of new properties and functions. The combination of metal and semiconductor renders the emergence of complementary optoelectronic properties that can be employed in potential technological applications in physics, chemistry, biology and medicine. Because many metal nanoparticles are embedded in single giant semiconductor architecture, these microstructures provide new model systems for the modification of respective theoretical models with many body interfacial electron transfer processes. Therefore, the findings of this research will help in developing a strategy that is capable of producing micro/nanostructures with the desired geometric features and specific functionalities.

\section{Conflicts of interest}

There are no conflicts to declare.

\section{Acknowledgements}

We gratefully acknowledge financial support from DST-SERB, New Delhi (Project No.: EMR/2016/006842). We are grateful to Prof. Yuichi Negishi, Tokyo University of Science, Shinjuku-ku, Japan, for providing support to X-ray Photoelectron Spectroscopy measurements.

\section{References}

1 Z. Zhang and J. T. Yates, Chem. Rev., 2012, 112, 5520-5551.

2 H. Harutyunyan, A. B. F. Martinson, D. Rosenmann, L. K. Khorashad, L. V. Besteiro, A. O. Govorov and G. P. Wiederrecht, Nat. Nanotechnol., 2015, 10, 770-774.

3 S. K. Cushing, J. Li, J. Bright, B. T. Yost, P. Zheng, A. D. Bristow and N. Wu, J. Phys. Chem. C, 2015, 119, 16239-16244.

4 T. Tatsuma, H. Nishi and T. Ishida, Chem. Sci., 2017, 8, 3325-3337.

5 W. Schottky, Z. Phys., 1939, 113, 367-414.

6 A. O. Govorov, H. Zhang, H. V. Demir and Y. K. Gun'ko, Nano Today, 2014, 9, 85-101.

7 S. K. Ghosh and T. Pal, Chem. Rev., 2007, 107, 4797-4862.

8 C. Voisin, D. Christofilos, P. A. Loukakos, N. Del Fatti, F. Vallée, J. Lermé, M. Gaudry, E. Cottancin, M. Pellarin and M. Broyer, Phys. Rev. B: Condens. Matter Mater. Phys., 2004, 69, 195416.

9 A. M. Brown, R. Sundararaman, P. Narang, W. A. Goddard III and H. A. Atwater, ACS Nano, 2016, 10, 957-966.

10 S. Link and M. A. El-Sayed, Int. Rev. Phys. Chem., 2010, 19, 409-453.

11 E. Blandre, D. Jalas, A. Petrov and M. Eich, ACS Photonics, 2018, 5, 3613-3620.

12 W. Li and J. G. Valentine, Nanophotonics, 2017, 6, 177-191.

13 B. A. Korgel, Nat. Mater., 2007, 6, 551-552.

14 U. Banin, Y. Ben-Shahar and K. Vinokurov, Chem. Mater., 2014, 26, 97-110.

15 R. Jiang, B. Li, C. Fang and J. Wang, Adv. Mater., 2014, 26, 5274-5309.

16 Z. L. Wang, Mater. Today, 2004, 7, 26-33.

17 C. Klingshirn, Chem. Phys. Chem., 2007, 8, 782-803. 
18 X. Liu, D. Li, X. Sun, Z. Li, H. Song, H. Jiang and Y. Chen, Sci. Rep., 2015, 5, 12555.

19 Y. Li, X. Zhao and W. Fan, J. Phys. Chem. C, 2011, 115, 3552-3557.

20 O. Volnianska, P. Boguslawski, J. Kaczkowski, P. Jakubas, A. Jezierski and E. Kaminska, Phys. Rev. B: Condens. Matter Mater. Phys., 2009, 80, 245212.

21 T.-J. Whang, M.-T. Hsieh and H.-H. Chen, Appl. Surf. Sci., 2012, 258, 2796-2801.

22 Y. Yan, M. M. Al-Jassim and S. H. Wei, Appl. Phys. Lett., 2006, 89, 181912.

23 M. He, Y. Tian, D. Springer, I. Putra, G. Xing, E. Chia, S. Cheong and T. Wu, Appl. Phys. Lett., 2011, 99, 222511.

24 B. P. Khanal, A. Pandey, L. Li, Q. Lin, W. K. Bae, H. Luo, V. I. Klimov and J. M. Pietryga, ACS Nano, 2012, 6, 3832-3840.

25 J. Wei, N. Jiang, J. Xu, X. Bai and J. Liu, Nano Lett., 2015, 15, 5926-5931.

26 T. N. Q. Trang, T. B. Phan, N. D. Nam and V. T. H. Thu, ACS Appl. Mater. Interfaces, 2020, 12, 12195-12206.

27 R. Wang, W. Yang, Y. Song, X. Shen, J. Wang, X. Zhong, S. Li and Y. Song, Sci. Rep., 2015, 5, 9189.

28 E. J. Guidelli, O. Baffa and D. R. Clarke, Sci. Rep., 2015, 5, 14004.

29 R. Sánchez Zeferino, M. Barboza Flores and U. Pal, J. Appl. Phys., 2011, 109, 014308.

30 J. K. Hyun, T. Kang, H. Baek, H. Oh, D.-S. Kim and G.-C. Yi, ACS Photonics, 2015, 2, 1314-1319.

31 A. Kim, Y. Won, K. Woo, C.-H. Kim and J. Moon, ACS Nano, 2013, 7, 1081-1091.

32 H. Y. Jun, C.-H. Chang, K.-S. Ahn, S. O. Ryu and C.-H. Choi, CrystEngComm, 2020, 22, 646-653.

33 H. R. Liu, G. X. Shao, Z. F. Zhao, Z. X. Zhang, Y. Zhang, X. Liang, X. G. Liu, H. S. Jia and B. S. Xu, J. Phys. Chem. C, 2012, 116, 16182-16190.

34 J. Manna, S. Goswami, N. Shilpa, N. Sahu and R. K. Rana, ACS Appl. Mater. Interfaces, 2015, 7, 8076-8082.

35 M. Zare, K. Namratha, S. Alghamdi, Y. H. E. Mohammad, A. Hezam, M. Zare, Q. A. Drmosh, K. Byrappa, B. N. Chandrashekar, S. Ramakrishna and X. Zhang, Sci. Rep., 2019, 9, 8303.

36 C. Pacholski, A. Kornowski and H. Weller, Angew. Chem., Int. Ed., 2002, 41, 1188-1191.

37 Z. Zheng, Z. S. Lim, Y. Peng, L. You, L. Chen and J. Wang, Sci. Rep., 2013, 3, 2434.

38 F.-R. Fan, Y. Ding, D.-Y. Liu, Z.-Q. Tian and Z. L. Wang, J. Am. Chem. Soc., 2009, 131, 12036-12037.

39 M. Ali, S. K. Pal, H. Rahaman and S. K. Ghosh, Soft Matter, 2014, 10, 2767-2774.

40 H. R. Liu, Y. Hu, Z. Zhang, X. Liu, H. Jia and B. Xu, Appl. Surf. Sci., 2015, 07, 644-652.

41 M. L. Kahn, T. Cardinal, B. Bousquet, M. Monge, V. Jubera and B. Chaudret, Chem. Phys. Chem., 2006, 7, 2392-2397.

42 X. Chen, Y. Li, X. Pan, D. Cortie, X. Huang and Z. Yi, Nat. Commun., 2016, 7, 12273.

43 M. Achermann, J. Phys. Chem. Lett., 2010, 1, 2837-2843.
44 F. Wu, L. Tian, R. Kanjolia, S. Singamaneni and P. Banerjee, ACS Appl. Mater. Interfaces, 2013, 5, 7693-7697.

45 S. K. Dutta, S. K. Mehetor and N. Pradhan, J. Phys. Chem. Lett., 2015, 6, 936-944.

46 Y. Dong, C. Feng, P. Jiang, G. Wang, K. Li and H. Miao, RSC Adv., 2014, 4, 7340-7346.

47 R. T. Tung, Appl. Phys. Rev., 2014, 1, 011304.

48 S. Lu, J. Qi, S. Liu, Z. Zhang, Z. Wang, P. Lin, Q. Liao, Q. Liang and Y. Zhang, ACS Appl. Mater. Interfaces, 2014, 6, 14116-14122.

49 J. Huang, Z. Yang, Z. Feng, X. Xie and X. Wen, Sci. Rep., 2016, 6, 24471.

50 M. E. Aguirre, H. B. Rodríguez, E. S. Román, A. Feldhoff and M. A. Grela, J. Phys. Chem. C, 2011, 115, 24967-24974.

51 Y. Min, M. Akbulut, K. Kristiansen, Y. Golan and J. Israelachvili, Nat. Mater., 2008, 7, 527-538.

52 R. B. Grubbs, Nat. Mater., 2007, 6, 553-555.

53 X. Yan, Z. Li, C. Zou, S. Li, J. Yang, R. Chen, J. Han and W. Gao, J. Phys. Chem. C, 2010, 114, 1436-1443.

54 Y. Liang, N. Guo, L. Li, R. Li, G. Ji and S. Gan, New J. Chem., 2016, 40, 1587-1594.

55 P. Fageria, S. Gangopadhyay and S. Pande, RSC Adv., 2014, 4, 24962-24972.

56 Y. Zheng, L. Zheng, Y. Zhan, X. Lin, Q. Zheng and K. Wei, Inorg. Chem., 2007, 46, 6980-6986.

57 N. Hasuike, R. Deguchi, H. Katoh, K. Kisoda, K. Nishio, T. Isshiki and H. Harima, J. Phys.: Condens. Matter, 2007, 19, 3652231.

58 J.-C. Valmalette, Z. Tan, H. Abe and S. Ohara, Sci. Rep., 2014, 4, 5238.

59 Y. Xie, S. Yang, Z. Mao, P. Li, C. Zhao, Z. Cohick, P.-H. Huang and T. J. Huang, ACS Nano, 2014, 8, 12175-12184.

60 Y. Yin, Y. Sun, M. Yu, X. Liu, T. Jiang, B. Yang, D. Liu, S. Liu and W. Cao, Sci. Rep., 2015, 5, 8152.

61 A. B. Djurisic, W. C. H. Choy, V. A. L. Roy, Y. H. Leung, C. Y. Kwong, K. W. Cheah, T. K. GunduRao, W. K. Chan, F. Lui and C. Surya, Adv. Funct. Mater., 2004, 14, 856-864.

62 S. Wang, F. Jia, X. Wang, L. Hu, Y. Sun, G. Yin, T. Zhou, Z. Feng, P. Kumar and B. Liu, ACS Omega, 2020, 5, 5209-5218.

63 M. D. L. R. Peralta, U. Pal and S. R. Zeferino, ACS Appl. Mater. Interfaces, 2012, 4, 4807-4816.

64 Y. Yao, F. Ji, M. Yin, X. Ren, Q. Ma, J. Yan and S. F. Liu, ACS Appl. Mater. Interfaces, 2016, 8, 18165-18172.

65 E. Chernysheva, W. Srour, B. Philippe, B. Baris, S. Chenot, R. F. Duarte, M. Gorgoi, H. Cruguel, H. Rensmo, H. Montigaud, J. Jupille, G. Cabailh, S. Grachev and R. Lazzari, Phys. Rev. B, 2018, 97, 235430.

66 https:/www.efunda.com/materials/piezo/material_data/mat data_output.cfm?Material.

67 K. Imazu, A. Yoshida, D. Tanaka and K. Tamada, SpringerPlus, 2014, 3, 284.

68 R. H. Fowler, Phys. Rev., 1931, 38, 45-56.

69 Y. Hattori, T. Taniguchi, K. Watanabe and K. Nagashio, ACS Appl. Mater. Interfaces, 2018, 10, 11732-11738. 